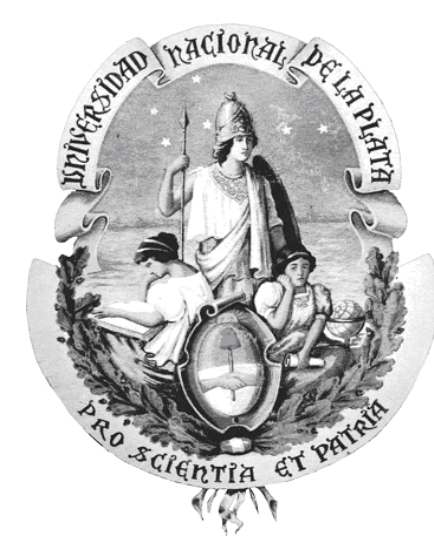

\title{
ESPACIOS VIRTUALES \\ PARA LA FORMACIÓN DE INVESTIGADORES ELEMENTOS DE ANALISIS Y DISEÑO
}

Tesista: Lic. Darío RODRIGUEZ

Director: Mg. Rodolfo BERTONE

Asesora Científica: Mg. María Florencia POLLO-CATTANEO

TESIS PRESENTADA PARA OBTENER EL GRADO DE MAGISTER

EN TECNOLOGIA INFORMATICA APLICADA EN EDUCACION

FACULTAD DE INFORMÁTICA

UNIVERSIDAD NACIONAL DE LA PLATA

Noviembre, 2011 


\section{AGRADECIMIENTOS}

A la Facultad de Informática de la Universidad Nacional de la Plata por acogerme con generosidad de "alma mater" para que pudiera realizar estudios de maestría en tecnología informática aplicada en educación.

Al Centro de Ingeniería del Software e Ingeniería del Conocimiento del Instituto Tecnológico de Buenos Aires por apoyarme en todas las instancias del proceso desarrollo de mi carrera de grado y en la etapa inicial de mi carrera de magister.

Al Grupo de Investigación en Sistemas de Información del Departamento de Desarrollo Productivo y Tecnológico de la Universidad Nacional de Lanús por recibirme para realizar la pasantía de investigación y desarrollo proveyendo un estimulante ambiente de intercambio de ideas con otros tesistas de postgrado, y apoyarme en todas las instancias del proceso desarrollo de mi carrera de docente investigador.

Al Mg. Rodolfo Bertone y a la Mg. María Florencia Pollo Cattaneo por dirigir mi trabajo de tesis, entrenándome en el pensamiento científico y guiándome en la aventura de la construcción de nuevo conocimiento, con dedicación de maestros y afecto de amigos.

Al Dr. Ramón García-Martínez, Director del Grupo de Investigación en Sistemas de Información por ser un estímulo permanente en mi formación como persona y profesional.

Al Ing. Pablo Pytel, Profesor Adjunto de la Cátedra de Inteligencia Artificial de la Facultad Regional Buenos Aires de la Universidad Tecnológica Nacional, por introducirme a la notación de predicados de orden n, revisando las primeras versiones de su uso en los diagramas de secuencia de dinámica grupal. 
A los Ingenieros Leonardo D'Onofrio y Fernando Uminsky del Laboratorio de Sistemas Inteligentes de la Facultad de Ingeniería de la Universidad de Buenos Aires, que desarrollaron el artefacto software utilizado para realizar la prueba de concepto del espacio virtual para la formación de investigadores a partir de las funcionalidades propuestas en la tesis. 